\title{
Reasoning with Imperfect Information and Knowledge
}

\author{
Gabriele Kern-Isberner ${ }^{1}$ - Igor Douven ${ }^{2}$ • \\ Markus Knauff ${ }^{3} \cdot$ Henri Prade $^{4}$
}

Nowadays, smart and intelligent computer systems are part of our everyday lives. However, computational intelligence is rather different from human intelligence, in particular, the problem of reasoning with uncertain, imprecise, incomplete, or inconsistent (in short, imperfect) information often renders computational methods relying on strict or deductive logics obsolete or fallacious whereas humans cope with this problem with relative ease. Reasoning with imperfect information plays a central role in practical deliberation and rational decision making. Indeed, models of human context-dependent reasoning that synthesise logical, philosophical and psychological aspects would provide novel insights into rational human reasoning. Several of such formal approaches have been developed in philosophy and artificial intelligence (AI) over the past decades, and an increasing interest in these new formal methods for rational human reasoning under uncertainty have emerged in psychology. Likewise, philosophers and computer scientists have shown an increased attention to the experimental methods of psychology recently. In particular for computer scientists and AI researchers, it is becoming more and

Gabriele Kern-Isberner

gabriele.kern-isberner@cs.tu-dortmund.de

Igor Douven

igor.douven@paris-sorbonne.fr

Markus Knauff

markus.knauff@psychol.uni-giessen.de

Henri Prade

prade@irit.fr

Technische Universität Dortmund, Dortmund, Germany

2 Paris-Sorbonne Université, Paris, France

3 Justus-Liebig-Universität Gießen, Gießen, Germany

4 Université Paul Sabatier, Toulouse, France 
more interesting to see whether the systems they have been developing are cognitively, respectively descriptively adequate. A synthesis of rational reasoning with imperfect information that takes into account research done in artificial intelligence, psychology and philosophy is needed for providing a clearer view of where we are and what are the pending issues both from computational resp. logical and cognitive viewpoints. This will give new impetus to formal, cognitive, and empirical models of rational reasoning, and as a side effect, might help making intelligent systems more helpful for their human users.

The idea for this special issue emerged from the seminar 15221: Multidisciplinary approaches to reasoning with imperfect information and knowledge-A synthesis and a roadmap of challenges which took place at Schloss Dagstuhl, Leibniz Center for Computer Science, May 26th-29th, 2015, and was organized by the editors of this special issue (for a report on this seminar, please see http://drops. dagstuhl.de/opus/frontdoor.php?source_opus=5403). This seminar brought together researchers interested in rational and uncertain reasoning from a very broad scientific scope to present and discuss problems and approaches from different disciplines, consolidate common grounds, and initiate new interdisciplinary collaborations. It took profit from the fact that computer scientists, philosophers, and psychologists have started quite recently to work in a common methodological paradigm with overlapping goals, converging interests, and largely shared research tools. The attendees identified challenges for new paradigms of rational reasoning, and discussed visions and foci for more interdisciplinary work. Most of the papers contained in this special issue were contributed by participants of this seminar and their colleagues.

Illusions in reasoning by Sangeet S. Khemlani and P.N. Johnson-Laird aims to show that human reasoners commit systematic fallacies, and that the theory of mental models can predict these errors. In particular, they point out that such fallacies are caused by the reasoners focussing on what is true in a possibility, but not what is false.

Uncertain reasoning is a core topic of this special issue, and probability theory is often considered to be the best formal tool for that. However, Tarek R. Besold, Artur d'Avila Garcez, Keith Stenning, Leendert van der Torre, and Michiel van Lambalgen make a claim for non-probabilistic methods in Reasoning in nonprobabilistic uncertainty: logic programming and neural-symbolic computing as examples. They describe and emphasise the benefits of logic-based methods like logic programming under Kleene semantics and a neural-symbolic implementation of Input/Output logic.

The high quality of non-probabilistic, even non-quantitative approaches to default logics for modelling human reasoning is also the topic of Formal nonmonotonic theories and properties of human defeasible reasoning by Marco Ragni, Christian Eichhorn, Tanja Bock, Gabriele Kern-Isberner, and Ping Ping Tse. The authors focus on conditionals as crucial entities of human reasoning and machine intelligence. From both a formal and an empirical perspective, they analyze systematically the power of formal nonmonotonic systems to model possible explicit defeaters, as in the Suppression Task, and more implicit conditional rules that trigger nonmonotonic reasoning by keywords. 
Niki Pfeifer and Leena Tulkki also deal with conditionals in their paper Conditionals, counterfactuals, and rational reasoning. An experimental study on basic principles. They present a unified approach for investigating rational reasoning about basic argument forms involving different forms of conditionals within coherence-based probability logic. In particular, they report on a new experiment showing that people interpret indicative conditionals and counterfactuals by coherent conditional probability assertions, and that they negate conditionals by negating their consequents.

In Stability and scepticism in the modelling of doxastic states: probabilities and plain beliefs, Hans Rott discusses two recent accounts of bridging the gap between plain logical belief and belief based on subjective probability, namely the Stability Theory of Hannes Leitgeb (2013-2015), and the Probalogical Theory (or Tracking Theory) of Hanti Lin and Kevin Kelly (2012), and he points out problems with these approaches.

Didier Dubois, Emiliano Lorini, Henri Prade present and discuss a logic of desires in The strength of desires: a logical approach. While there are some similarities to beliefs from a formal point of view, the authors argue that the way desires should be treated is very different from beliefs. They also consider revision of desires and again, point out parallels to and differences from belief revision. They suggest possibility theory as a suitable syntactic framework for encoding desire change.

Finally, Pierre Bisquert, Madalina Croitoru, Florence Dupin de Saint Cyr, and Abdelraouf Hecham address the benefits of argumentation theories in their paper Formalizing cognitive acceptance of arguments: Durum wheat selection interdisciplinary study. They propose a computational cognitive model for argument acceptance which is based on the dual model system in cognitive psychology, and apply this to an interdisciplinary project in an agronomy setting.

Acknowledgements First of all, we would like to thank Schloss Dagstuhl for hosting our seminar, providing us with an excellent service and the opportunity for having this scientific come together in the unique collaborative and inspiring atmosphere of the castle. Without this seminar, this special issue would not have been possible, and we are also grateful to all attendees of the seminar for sharing their ideas with us. The idea for this seminar arose from activities of the DFG Priority Program 1516 New frameworks of rationality which was initiated by Markus Knauff; the pleasure we have been experiencing with interdisciplinary work and fruitful collaborations within this Priority Program have encouraged us to discuss our research work and future goals with a broader audience. We are also thankful to all authors of this issue who provided with their papers a proof that the Dagstuhl seminar was not just one inspiring week but also had long-lasting effects. Gregory Wheeler was one of the participants of that seminar, and he was the first to have the idea of this special issue, so our special thanks go to him, and also to the current editor-in-chief Mariarosaria Taddeo whose help with finalizing this issue is very much appreciated. We also thank all reviewers of the submitted papers for delivering reviews of an excellent quality in time. 